\title{
Recent advances in Earth remote sensing: Fourier Transform Stationary HyperSpectral Imagers
}

\author{
Alessandro Barducci, Paolo Marcoionni and Ivan Pippi \\ Istituto di Fisica Applicata «Nello Carrara» (IFAC), CNR, Firenze, Italy
}

\begin{abstract}
Future trends for the development of new remote sensing imagers have being defined since the launch of the first Fourier Transform HyperSpectral Imager (FTHSI) on board of DoD technological satellite MightySat II.1. Starting from the analysis of FTHSI optical configuration we have proposed an interesting modification which produces an image of the observed surface superimposed to a stationary interference pattern. This new optical arrangement together with the possibility to accommodate the spectral resolution by changing the device optical aperture and the sensor sampling step make the new instrument interesting for Earth remote sensing purposes. In this paper we present some preliminary results obtained from a laboratory prototype developed at our Institute. Some hints are discussed about the use of such an instrument on board of airborne and satellite platforms.
\end{abstract}

Key words Fourier transform imaging spectrometer - stationary Sagnac interferometer - spectral calibration - hyperspectral remote sensing

\section{Introduction}

Recent advances in optics technology and on-board pre-elaboration data facilities have driven the attention of the international scientific community towards the development of the so called «stationary imaging interferometers» for Earth remote sensing purposes (Junttila, 1992; Horton, 1996). These interferometers do not employ any moving part to scan the imaged Field of View (FoV) and generate the entire interference pattern simply moving over the surface of the observed target (Junttila, 1991; Descour, 1996; Horton et al., 1997).

Mailing address: Dr. Ivan Pippi, Istituto di Fisica Applicata «Nello Carrara» (IFAC), CNR, Via Madonna del Piano 10, 50019 Sesto Fiorentino (FI), Italy; e-mail: I.Pippi@ifac.cnr.it
The launch of the first Fourier Transform HyperSpectral Imager (FTHSI) on board of US Department of Defence (DoD) technological satellite MightySat II.1 was an attempt to overcome the main drawbacks that limit the use of push-broom and whisk-broom imaging spectrometers for environment investigation (Meigs et al., 1997; Otten III et al. 1997, 1998).

These limitations concern sensor calibration, electronics complexity and the circumstance that spectral parameters such as spectral resolution and sampling step cannot be changed during the flight.

System precursors of FTHSI had been the HyperCam and the IrCam developed by Kestrel Corporation (U.S.A.) for airborne applications, and the Spatially Modulated Fourier Transform Spectrometer (SMIFTS) developed by Hawaii University. The main properties of these instruments are listed in table I.

In a stationary interferometer all the impinging radiation is collected by the detector for interferogram sampling (Jacquinot and Fellgett advantages) (Jacquinot, 1954; Bennett et al., 1993; Persky, 1995). This is a significant im- 
Table I. The main properties of FTHSI and its precursors.

\begin{tabular}{ccccc}
\hline \hline Instrument & FTHSI & HyperCam & IrCam & SMIFTS \\
Spectral range & $350-1050 \mathrm{~nm}$ & $450-1050 \mathrm{~nm}$ & $1700-5000 \mathrm{~nm}$ & $1000-5200 \mathrm{~nm}$ \\
& & & & $3000-5000 \mathrm{~nm}$ \\
Spectral resol. & $85.4 \mathrm{~cm}^{-1}$ & $87 \mathrm{~cm}^{-1}$ & $45 \mathrm{~cm}^{-1}$ & $95 \mathrm{~cm}^{-1}$ \\
& $(1.7 \mathrm{~nm} @ 450 \mathrm{~nm})$ & & $(50 \mathrm{~nm} @ 3000 \mathrm{~nm})$ & $35 \mathrm{~cm}^{-1}$ \\
Channels & 256 & 180 & 55 & 256 \\
Field-of-view & $1.75^{\circ}$ & $13^{\circ}$ & $14^{\circ}$ & $13^{\circ}$ \\
Altitude & $570 \mathrm{~km}$, & $3 \mathrm{~km}$ & $3 \mathrm{~km}$ & \\
& Sun-synchronous orbit & & &
\end{tabular}

provement of the Signal-to-Noise Ratio (SNR) because all the photons coming from a pixel of the source contribute to the interferogram power, no matter what their wavelength is. On the contrary, filters, gratings and prisms of a standard spectrometer reject most of the electromagnetic radiation field. The principal critical points of an imaging interferometer are connected with the spreading of the observed target pixels originated by the not-entirely compensated instrument motion, and the high data-rate requested.

Starting from the analysis of FTHSI optical configuration we have developed a laboratory prototype of the imaging interferometer.

In Section 2 we briefly discuss the adopted optical configuration and the experimental activity we have performed in order to calibrate the instrument response. In Section 3 we give our conclusions.

\section{Experimental activity}

The laboratory device uses a new image plane interferometer geometry to produce «autocorrelation function modulation» in the image plane of the two dimensional array such as the phase offset of the modulation linearly varies across the images. The interferogram is imaged onto a CCD array as a spatial distribution of intensity rather than as a function of time. The typical optical layout of the developed interferometer, called Sagnac configuration, is shown in fig. 1.

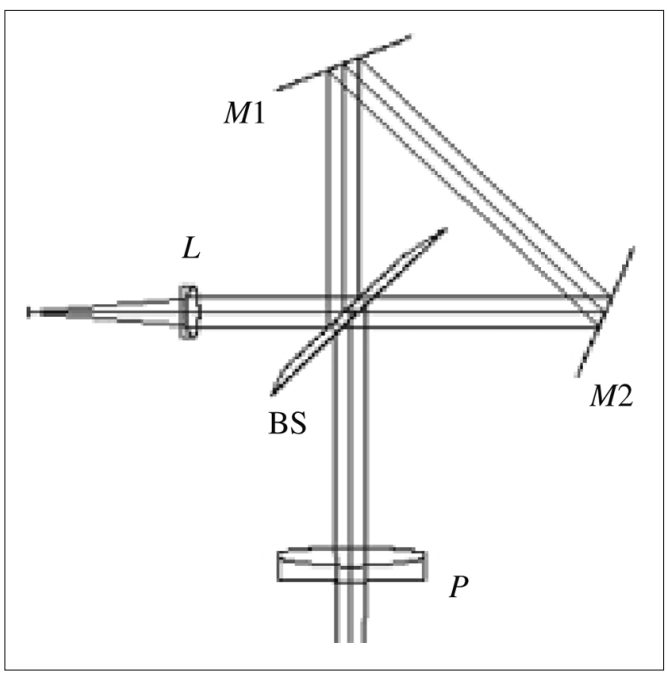

Fig. 1. Layout of the stationary interferometer arranged in the triangular geometry. The light from the object is first collimated by the objective $L$, it enters the device, it exits from it at $90^{\circ}$ though two folding mirrors $(M 1$ and $M 2)$ after being split into two coherent beams by the Beam-Splitter BS put at $45^{\circ}$ with respect to the optical axes. $P$ collocates the lens which focuses the energy on the CCD plane.

The characteristics of the CCD used to spatially sample the interferogram are listed in table II.

As a two dimensional image is formed by the fore-optics, an entire frame is recorded for each autocorrelation phase offset. The three dimensional array of this data is processed to generate 
Table II. Principal characteristics of the CCD array used to sample the interferogram.

$\begin{array}{cc}\text { Type } & \text { CCD frame-transfer with anti-blooming, TH7887A } \\ \text { Number of pixel } & 1024 \times 1024 \\ \text { Pixel size } & 14 \mu \mathrm{m} \times 14 \mu \mathrm{m} \\ \text { Spectral range } & 430 \mathrm{~nm}-1000 \mathrm{~nm} @ \mathrm{QE}>3 \% \\ \text { Responsivity } & 23 \mathrm{DN} /\left(\mathrm{nJ} \mathrm{cm}^{2}\right) @ 450 \mathrm{~nm} \\ & 18 \% @ 680 \mathrm{~nm} \\ \text { Dynamic range } & 3200: 1 \\ \text { Maximum frame/rate } & 60 \mathrm{fps} \\ \text { Digitalization } & 12 \mathrm{bit}\end{array}$

an autocorrelation function data-cube which is Fourier transformed to yield a wavenumber hyperspectral data-cube.

The fundamental law, which describes the intensity $I(\mathrm{OPD})$ falling on the photosensitive element (neglecting the optical losses) is

$$
I(\mathrm{OPD})=\int \frac{I_{0}(k)}{2}[1+\cos (2 \pi k \mathrm{OPD})] d k
$$

where $I_{0}(\lambda)$ is the intensity of the ray before entering the interferometer, OPD is the optical path difference introduced by the beam splitter for every direction of propagation, and $k=1 / \lambda$ is the wavenumber relative to $\lambda$. As can be seen from eq. (2.1), the interferogram is an oscillating function which has a maximum at $\mathrm{OPD}=0$ and decays for large OPD's.

Actually, an interferogram is constituted by a pair of values $\xi, \mathrm{DN}(\xi)$ which respectively indicate the pixel position on the matrix and the corresponding electronic signal expressed in digital number. As shown in eq. (2.2), the spatial coordinate $\xi$ of the pixel can be linked to the sampling step determined as the distance $p$ (pitch) between the centres of two adjacent pixels

$$
\xi=\left(j-j_{0}\right) p
$$

$j$ being the index of the acquired interferogram and $j_{0}$ the position of the pixel corresponding to the null OPD. As we have stated, the relation between OPD and the propagation direction $\vartheta$ of a single ray entering the interferometer is linear, as long as the imaged FoV is small enough

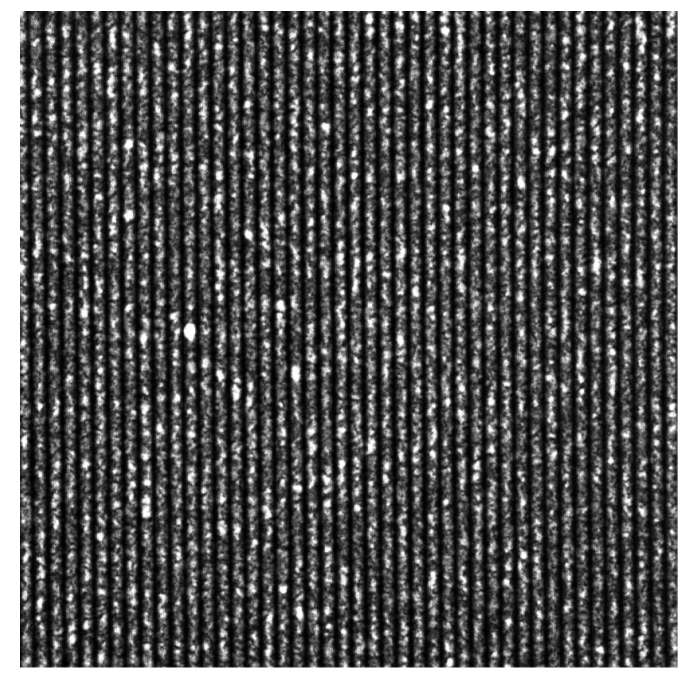

Fig. 2. Raw (level 0) image (in gray-scale) obtained illuminating a double planar diffuser with a $\mathrm{He}-\mathrm{Ne}$ laser. We observe a pattern of vertical fringes superimposed to the target image.

$$
\operatorname{OPD}(\vartheta)=a \vartheta=\frac{a}{f} \xi=\frac{a}{f}\left(j-j_{0}\right) p
$$

$f$ being the focal length of the lens which focuses the interference image, and $a$ expresses the direct proportionality between OPD and $\vartheta$. In order to calibrate in wavenumber (or in OPD values) the response of the interferometer we employed a He-Ne laser $\left(\lambda_{\text {exc }}=632.8 \mathrm{~nm}\right)$ illuminating a double planar diffuser to produce uniform (homoge- 
neous and isotropic) radiation field at the instrument entrance. Figure 2 shows a single imageframe obtained with the laser source.

As can be seen, the instrument FoV is entirely filled with straight stripes (also called «fringes of equal thickness»), due to electromagnetic interference overlapping the flat input image. The shape of this pattern is due to the circumstance that all pixels on a vertical line go through the same OPD, so that they undergo the same amount of interference for the same wavelength of light. Conversely, the high number of these fringes is due to the high intrinsic coherence-degree of the employed source.

The image here presented was pre-processed according to the following scheme:

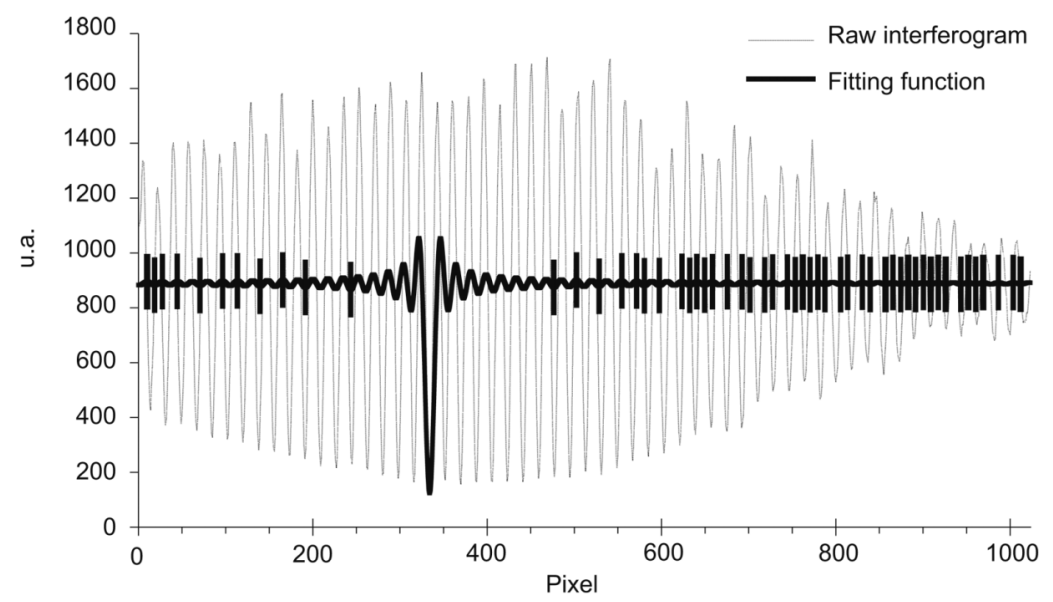

Fig. 3. Interferogram acquired onto the $\mathrm{CCD}$ plane relative to an impulse-like source, having a bandwidth far below the spectral resolution of the imaging system. The employed source is a He-Ne laser. The solid line indicated the fitting function used to retrieve the pixel position corresponding to the null OPD.

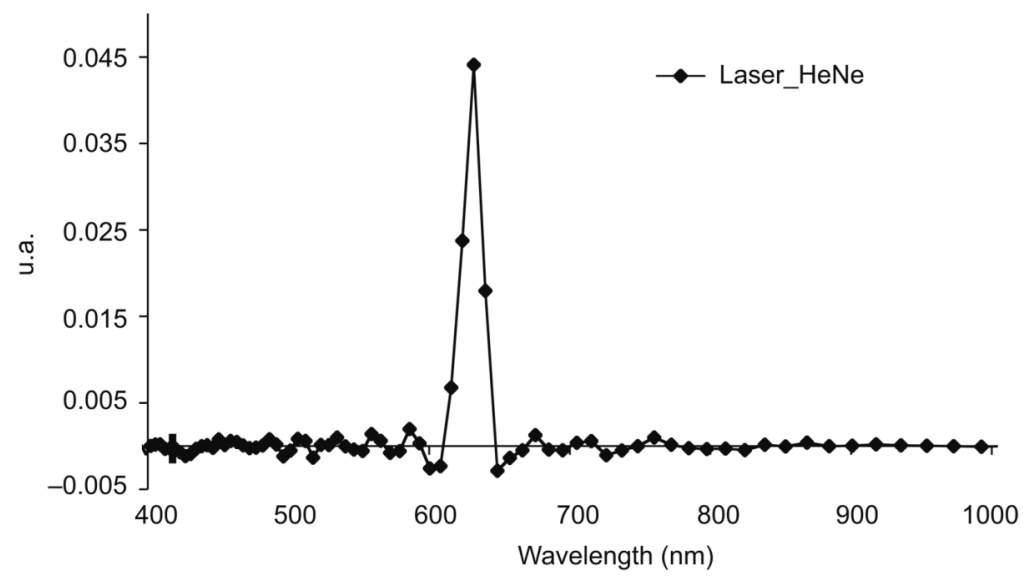

Fig. 4. Un-calibrated spectral radiance retrieved from the interferogram shown in fig. 3 after offset subtracting. 
- dark signal subtraction;

- instrument spatial response compensation;

- geometrical distortion correction (vignetting, spatial shift of the fringes pattern).

Then, from the single frame an interferogram averaged over all pixels was extracted. Its central part was fitted with a sinc-like function in order to estimate and subtract the DC offset as expressed in eq. (2.1) by the term $I_{0}(k) / 2$. An ideal interferogram is symmetric with respect to zero path difference and contains cosine contributions only.

However, additional phase errors caused by the misaligned sampling grid, which does not match the region of zero path difference, result in an evident asymmetry in a real interferogram shape.

Figure 3 shows an average interferogram and the fitting function, which best fits its central part. In this plot a strong asymmetry is evident with respect to the pixel scale.

After offset subtracting, the new interferogram is Fourier transformed to retrieve the uncalibrated spectral radiance of the employed source. Figure 4 shows the result of inverse cosine transform obtained without adopting any apodization.

\section{Conclusions}

In this paper we have presented preliminary images acquired with a new stationary imaging interferometer operating in Sagnac configuration. We have discussed the experimental activity carried out in order to calibrate the interferometer response. In addition we have analyzed a methodology to pre-process the acquired raw interferogram for dark-signal subtraction, instrument spatial response compensation and geometrical distortion correction (vignetting, spatial shift of the fringes pattern). Future investigations will address the option to employ this device on board of an airplane for Earth remote sensing purposes.

\section{REFERENCES}

Bennet, C.L., M. Carter, D. Fields and J. Hernandez (1993): Imaging Fourier transform spectrometer, SPIE Proc., 1937, 191-200.

Cabib, D., R.A. Buckwald, Y. Garini and D.G. Soenksen (1996): Spatially resolved Fourier transform spectroscopy (spectral imaging): a powerful tool for quantitative analytical microscopy, SPIE Proc., 2678, 278291.

Descour, M.R. (1996): The throughput advantage in imaging Fourier-transform spectrometers, SPIE Proc., 2819, 285-290.

HorTON, R.F. (1996): Optical design for a high-Etendue imaging Fourier transform spectrometer, SPIE Proc., 2819, 300-315.

Horton, R.F., C.A. Conger and L.S. Pellegrino (1997): High Etendue imaging Fourier transform spectrometer - Initial results, SPIE Proc., 3118, 380-390.

JACQUINOT, P. (1954): The luminosity of spectrometers with Prisms, Grating, or Fabry-Perot Etalons, J. Opt. Soc. Am., 44, 761-765.

JunTtilA, M.L. (1991): Performance limits of stationary Fourier spectrometers, J. Opt. Soc. Am., A8, 14571462.

JunTtila, M.L. (1992): Stationary Fourier transform spectrometer, Appl. Opt., 31, 4106-4112.

Meigs, A.D., L.J. Otten III, T.Yu. Cherezova, J.B. Rafert and R.G. SELLAR (1997): LWIR and MWIR ultraspectral Fourier transform imager, SPIE Proc., 3221, 421428.

Otten III, L.J., A.D. Meigs, B.A. Jones, P. Prinzing, S. Don Fronterhouse, R.G. Sellar, J.B. Rafert and C. Hodge (1997): The engineering model for the MightySat II.1 hyperspectral image, SPIE Proc., 3221, 412420.

Otten III, L.J, A.D. Meigs, B.A. Jones, P. Prinzing and S. Don Fronterhouse (1998): Payload qualification and optical performance test results for the MightySat II.1 hyperspectral imager, SPIE Proc., 3498, 231-238.

PERSKY, M.J. (1995): A review spaceborne infrared Fourier transform spectrometer for remote sensing, Rev. Sci. Instrum., 66 (10), 4763-4797. 